\title{
Proline-Independent Binding of PUT3 Transcriptional Activator Protein Detected by Footprinting In Vivo
}

\author{
JEFFREY D. AXELROD, ${ }^{1}$ JOHN MAJORS, ${ }^{1 *}$ AND MARJORIE C. BRANDRISS ${ }^{2}$ \\ Department of Biochemistry and Molecular Biophysics, Washington University School of Medicine, \\ St. Louis, Missouri $63110,{ }^{1}$ and Department of Microbiology and Molecular Genetics, University of \\ Medicine and Dentistry of New Jersey-New Jersey Medical School, Newark, New Jersey 07103 ${ }^{2}$
}

Received 7 June 1990/Accepted 20 September 1990

\begin{abstract}
The PUT3 gene product is a transcriptional activator required for expression of the enzymes of the proline utilization pathway. Using two methods of footprinting in vivo, we have determined that PUT3 protein is poised at the promoters of the genes encoding these enzymes and that proline-mediated induction modulates the activity of constitutively bound PUT3.
\end{abstract}

The PUT3 gene product (referred to as PUT3) is a transcriptional activator of $P U T 1$ and $P U T 2$, the structural genes for the enzymes of the proline utilization pathway $(5,24)$. PUT3 binds short sequences found in the promoters of $P U T 1$ and PUT2 (23, 24), inducing 50- and 15-fold (respectively) increases in expression when proline is the sole nitrogen source $(4,6,28,29)$. In vitro studies using gel mobility shift assays demonstrated that PUT3, either by itself or as part of a complex, bound to these promoters in the presence or absence of proline in the culture medium (24). These studies did not determine whether the binding of PUT3 is regulated in vivo.

Yeast transcriptional activators that induce expression of regulated genes may be divided into two categories. The first consists of DNA-binding proteins that are bound to their recognition sites only when the target gene is on (e.g., GCN4 [11], HAP1 [18], and ACE1 [9, 26]). The second category is made up of activators that are constitutively bound to the DNA but whose activity is modulated (e.g., ADR1 [7, 8, 27], HSTF [25], and GAL4 in the absence of catabolite repression $[10,15,22])$. The mechanisms by which any of these proteins stimulate transcription are not well understood (12, 19, 20). The studies described here used two methods of footprinting in vivo to demonstrate that PUT3 is poised at the PUT1 and PUT2 promoters and thus belongs in the second category.

The first footprinting method relies on the principle that the formation of UV light-induced photodimers is dependent on the conformation of the DNA, which is in turn sensitive to the presence of bound proteins $(1,2,21,30)$. The second method detects protection of protein-bound DNA from methylation by dimethyl sulfate (DMS) in vivo (1). The footprinting methods have been previously reported (1) and were used with minor modifications. Briefly, yeast cultures were grown in the appropriate media, harvested, and resuspended in phosphate-buffered saline. For photofootprinting, they were then exposed to UV light under a transilluminator for $60 \mathrm{~s}$, and DNA was isolated as described previously (1). PUTI samples were cut with HaeIII, and PUT2 samples were cut with EcoRI. The DNA was then adjusted to 0.5 $\mu \mathrm{g} / \mu \mathrm{l}$ by using a spectrophotometer, and $3.5 \mu \mathrm{g}$ of DNA was used per primer extension reaction. After electrophoresis, sequencing gels were fixed and dried for autoradiography.

\footnotetext{
* Corresponding author.
}

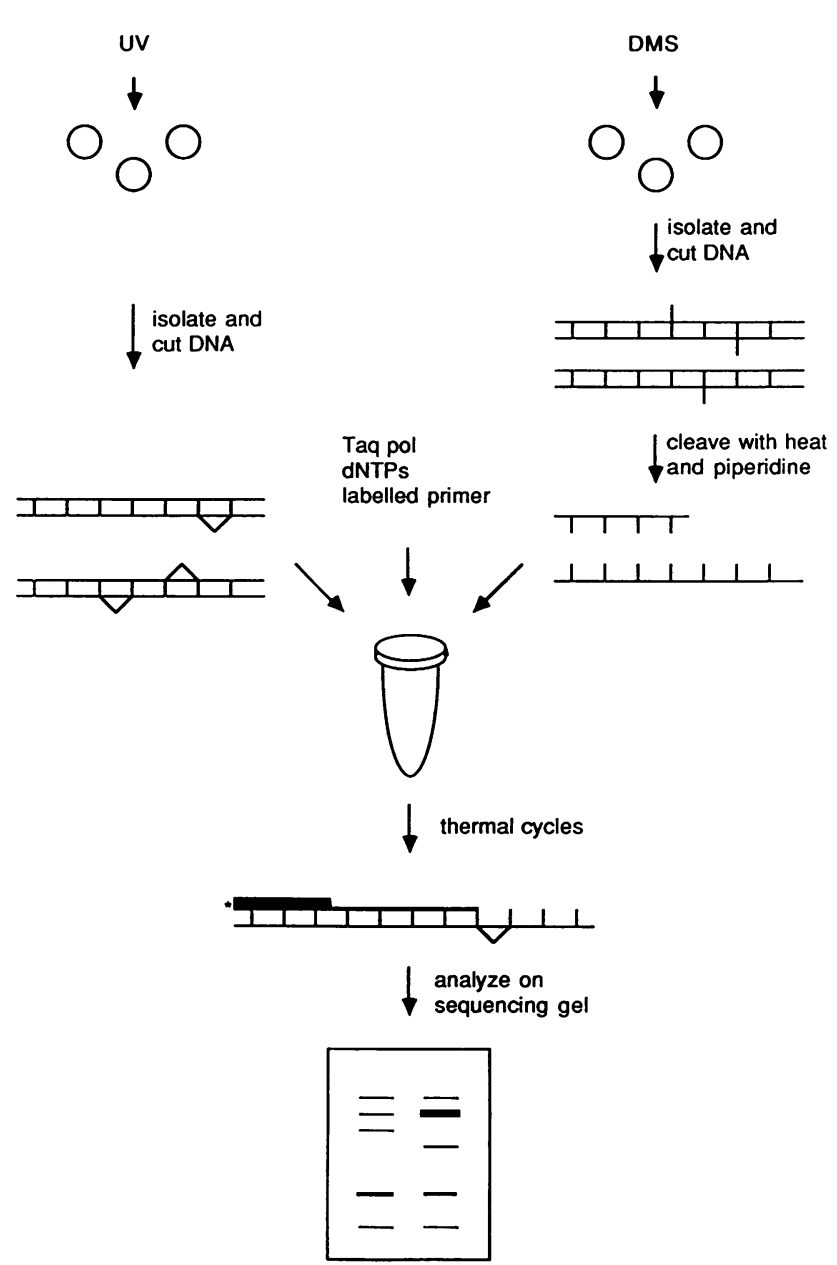

FIG. 1. Summary of footprinting methods. Intact yeast cells are irradiated with UV light under conditions of interest. Then, using whole yeast genomic DNA as a template, the photoproducts are mapped and quantitated by multiple rounds of primer extension with Taq polymerase, which is blocked by photoproducts in the template strand. Primer extension products are then detected on a sequencing gel, where either enhancements or repressions of bands can indicate the presence of a bound protein. The second method uses the same assay to detect protection of the DNA from methylation by DMS in vivo (1). dNTPs, Deoxynucleoside triphosphates. 


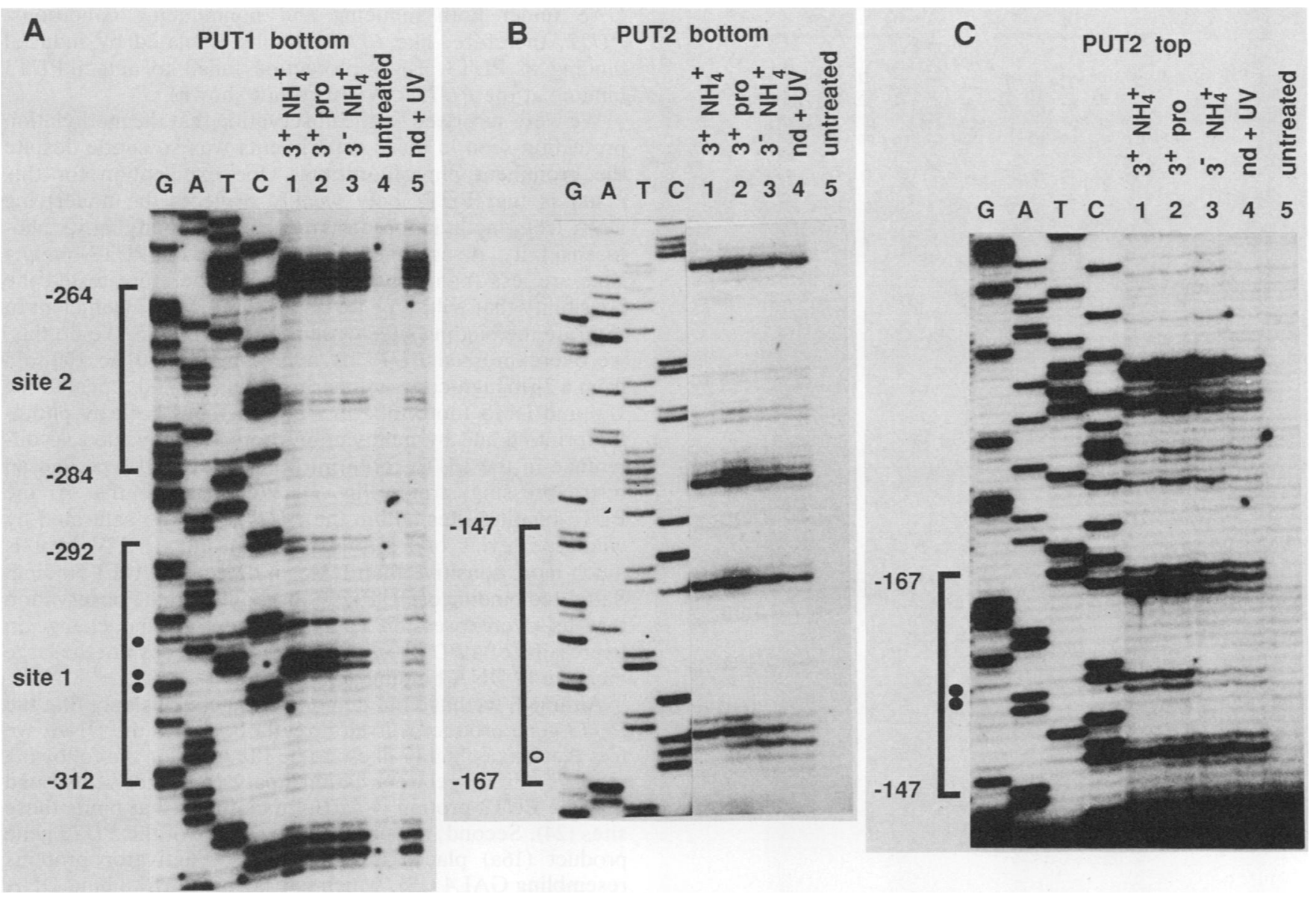

FIG. 2. (A) Footprinting of the PUTI UAS. Strains used were either wild type for PUT3 $\left(3^{+}\right)$(MB758-1C [MAT $\alpha$ ura3-52 trpl PUT3]) or null for PUT3 $\left(3^{-}\right)$(JM5010 [MAT $\alpha$ ura3-52 trpl put3-4::TRP1]). The put3-4::TRPl allele (16a) deletes all but the last 13 codons of the PUT3 open reading frame. Cultures were grown on ammonium sulfate $\left(\mathrm{NH}_{4}{ }^{+}\right)$or proline (pro) as the sole nitrogen source in a medium containing $0.17 \%$ yeast nitrogen base, $2 \%$ glucose, and either $0.2 \%$ ammonium sulfate or $0.1 \%$ proline. Uracil and tryptophan were supplemented as required. Untreated DNA (untreated) or naked DNA irradiated in vitro (nd + UV) were subjected to the primer extension reactions as controls. The PUT3 consensus sites are marked by brackets, and their positions are indicated (28). PUT3-dependent enhancements in photoproduct formation are indicated (๑). An additional enhancement was seen at position -291 in other experiments but is not seen clearly in this gel. The bottom-strand oligonucleotide spanned from -392 to -374 (with respect to the +1 at ATG) (28) and was annealed at $50^{\circ} \mathrm{C}$. All footprint experiments were repeated several times, and only changes that were most consistently and reproducibly observed are marked. (B and C) Bottom- and top-strand UV footprints of the PUT2 UAS. O, PUT3-dependent photoproduct repression. The single PUT3-binding site is marked with a bracket. All other symbols are as described above. The bottom-strand PUT2 oligonucleotide spanned from -259 to -242 , and the top-strand oligonucleotide spanned from -57 to -79 (23). Both were annealed at $50^{\circ} \mathrm{C}$. Interestingly, PUT3 binding did not induce photoproduct formation at equivalent positions in the three binding sites. This may be a result of photofootprint changes being dependent on features of the binding sites that are not conserved among the three sites.

Naked DNA samples were isolated from unirradiated cells and were irradiated in vitro for $5 \mathrm{~s}$. For DMS footprinting, the cultures were suspended in phosphate-buffered saline and treated with DMS as described previously (1). DNA was isolated and digested, cleaved in $1 \mathrm{M}$ piperidine at $90^{\circ} \mathrm{C}$, lyophilized, resuspended in $200 \mu$ l of $\mathrm{H}_{2} \mathrm{O}$, transferred to new tubes, and precipitated with $3 \mu \mathrm{l}$ of $1 \mathrm{M}$ spermidine. The pellets were resuspended in $300 \mu$ l of $3 \mathrm{M}$ ammonium acetate at $68^{\circ} \mathrm{C}$, ethanol precipitated, resuspended in $100 \mu$ l of $\mathrm{H}_{2} \mathrm{O}$, and lyophilized again. The final pellets were resuspended in $\mathrm{H}_{2} \mathrm{O}$, transferred to new tubes, and adjusted to $0.5 \mu \mathrm{g} / \mu \mathrm{l}$. Samples were then treated as described above. The approach is outlined in Fig. 1.

We photofootprinted in vivo a region of the $P U T 1$ promoter that was previously determined to function as the upstream activation sequence (UAS) and which contains two PUT3-binding sites (24). PUT3 binding could be detected in vivo by comparing a wild-type strain grown under inducing conditions with a strain bearing a null $\Delta p u t 3$ allele (Fig. 2A, lanes 2 and 3 ). Within the bottom strand of PUT3-binding site 1, several bands, representing photoproducts, were of greater intensity in the induced wild-type strain than in both the $\Delta p u t 3$ strain and irradiated, naked DNA (Fig. 2A, lane 5). These changes in the photoproduct pattern are indicative of PUT3-dependent binding to this site. We believe that PUT3 protein binds directly to this site, and we will present arguments to support this contention below.

An uninduced wild-type strain yielded a photoproduct pattern resembling that of an induced wild-type strain, indicating that the PUT3 protein is bound to this site even under conditions in which the gene is not expressed. PUT3binding site 2 on the bottom strand and both sites on the top 


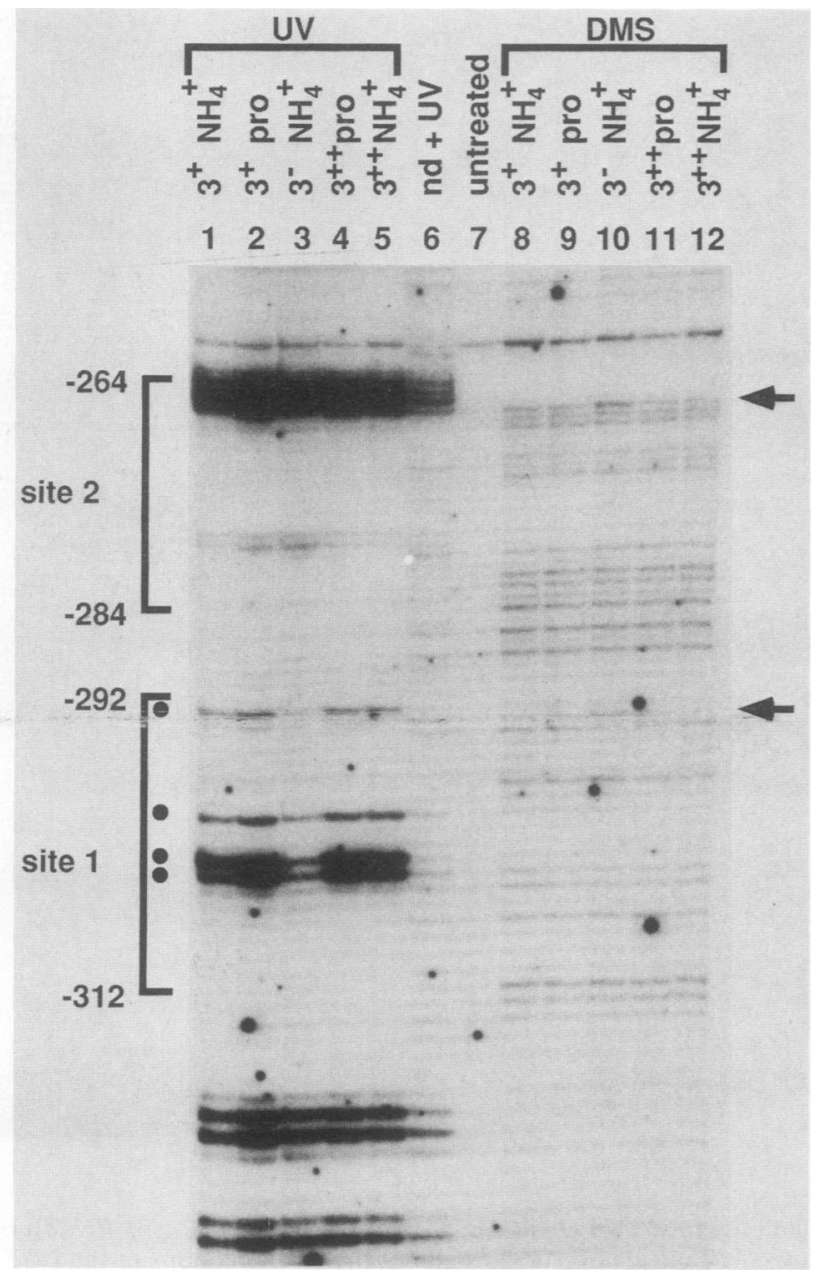

FIG. 3. Footprints of the PUTI UAS in strains with wild-type or overexpressed levels of PUT3. Strains were wild type at PUT3 $\left(3^{+}\right)$ or overexpressed $P U T 3$ from a $2 \mu \mathrm{m}$ plasmid $\left(3^{++}\right)$. MB758-1C was made to overexpress PUT3 by transformation with plasmid pDB37, a $2 \mu \mathrm{m}$ plasmid bearing an $11-\mathrm{kb}$ fragment containing the PUT3 gene (16). All other symbols are as in Fig. 2. Additional cultures were footprinted with DMS. Cultures were suspended in phosphatebuffered saline and treated with DMS as described in the text (see Fig. 1). The arrows mark sites of PUT3-dependent methylation protection.

strand had no photoproduct targets that were reproducibly altered in intensity by PUT3 binding (data not shown).

Additional cultures were footprinted with DMS in vivo (Fig. 3, lanes 8 to 12). Two subtle repressions, one in each PUT3-binding site, could be seen by comparison of an induced wild-type strain with a $\Delta p u t 3$ strain. These footprints were quite weak but were evident in three independent experiments (data not shown). While the methylation protection results are consistent with the observation that PUT3 binding is not regulated by proline, comparison of the DMS and UV light footprints demonstrates the much greater sensitivity of photofootprinting in detecting PUT3 binding.

We also footprinted the single PUT3-binding site within $P U T 2$, using both light and DMS as probes (Fig. 2B and C). Both the top and bottom strands of the PUT2 UAS revealed PUT3-dependent photofootprint changes. The photofootprints of both strands indicate that PUT3 is bound to the
UAS under both inducing and noninducing conditions. $P U T 2$, therefore, like $P U T 1$, is not regulated by induced binding of PUT3. DMS protection failed to detect PUT3 binding at the PUT2 UAS (data not shown).

We were surprised by the observation that the methylation protection seen in these experiments was so subtle despite the prominent photofootprints. One explanation for this result is that PUT3 only weakly protects the underlying DNA from methylation despite strongly modifying its photosensitivity. A second explanation is that the PUT3-binding sites are less than fully occupied. We therefore tested the possibility that wild-type levels of PUT3 were insufficient to saturate the binding sites within the PUTI UAS. To do this, we overexpressed PUT3 by approximately 50- to 100 -fold from a $2 \mu \mathrm{m}$ high-copy-number plasmid (24) and repeated the UV and DMS footprints on PUT1. Assays both by photofootprinting and by methylation protection revealed no difference in the PUT3 footprint between a wild-type and an overexpressing strain (Fig. 3). We conclude that (i) the PUT3-binding sites within the PUT1 UAS are saturated by wild-type levels of PUT3 expression and (ii) UV light is much more sensitive than DMS in detecting PUT3 binding. Saturated binding of PUT3 is consistent with the observation that its overexpression leads to essentially no change in expression of a PUT2-lacZ fusion gene (16) despite the large increase in DNA-binding activity (24).

Although we have not demonstrated conclusively that the PUT3 gene product is itself directly bound to the DNA, we feel that this is highly likely to be the case for the following reasons. First, gel retardation analysis has demonstrated that the PUT3 protein is part of a complex that binds these sites (24). Second, the predicted sequence of the PUT3 gene product (16a) places it in a class of activator proteins resembling GAL4 (13), which has been shown to bind DNA directly (3) via a zinc cluster motif $(14,17)$.

Since both the transcription $(16,16 a)$ and in vivo DNA binding (this work) of PUT3 are independent of the presence of proline in the medium, activation of the proline utilization genes must involve changes in activity of the DNA-bound PUT3 protein. Several mechanisms can be envisioned. (i) PUT3 itself might bind proline, resulting in a change in its conformation and thus its contacts with proteins of the transcription apparatus. (ii) A proline-induced covalent modification of PUT3 could alter its activity. (iii) PUT3 may interact with another protein that serves to repress its activity in the absence of proline or potentiate its activity in the presence of proline. Current work is aimed at resolving this issue.

We note that the PUT3 footprints detected by UV light are substantially more pronounced than are those detected by methylation protection in this study. In this case, photofootprinting is clearly the more sensitive method. This is in contrast to results obtained with GAL4, where we see equally robust UV and methylation protection footprints in at least several binding sites (1). Clearly, the use of multiple footprinting probes should increase the probability of detecting a protein-DNA interaction of interest. To our knowledge, UV light and DMS are the only probes that have been described for use in mapping these interactions in unbroken cells.

This work was supported by a National Research Service Award, Medical Scientist, GM07200 (J.D.A.), by National Institutes of Health Public Health Service grant R01 GM40751 (M.C.B.), and by the Washington University-Monsanto agreement (J.M.). 


\section{REFERENCES}

1. Axelrod, J. D., and J. Majors. 1989. An improved method for photofootprinting yeast genes in vivo using Taq polymerase. Nucleic Acids Res. 17:171-183.

2. Becker, M. M., and J. C. Wang. 1984. Use of light for footprinting DNA in vivo. Nature (London) 309:682-687.

3. Bram, R. J., and R. D. Kornberg. 1985. Specific protein binding to far upstream activating sequences in polymerase II promoters. Proc. Natl. Acad. Sci. USA 82:43-47.

4. Brandriss, M. C. 1983. Proline utilization in Saccharomyces cerevisiae: analysis of the cloned PUT2 gene. Mol. Cell. Biol. 3:1846-1856.

5. Brandriss, M. C. 1987 . Evidence for positive regulation of the proline utilization pathway in Saccharomyces cerevisiae. Genetics 117:429-435.

6. Brandriss, M. C., and B. Magasanik. 1979. Genetics and physiology of proline utilization in Saccharomyces cerevisiae: enzyme induction by proline. J. Bacteriol. 140:498-503.

7. Cherry, J. R., T. R. Johnson, C. Dollard, J. R. Schuster, and C. L. Denis. 1989. Cyclic AMP-dependent protein kinase phosphorylates and inactivates the yeast transcriptional activator ADR1. Cell 56:409-419.

8. Denis, C. L., and C. Gallo. 1986. Constitutive RNA synthesis for the yeast activator ADR1 and identification of the $A D R 1-5^{c}$ mutation: implications in posttranslational control of $A D R 1$. Mol. Cell. Biol. 6:4026-4030.

9. Furst, P., S. Hu, R. Hackett, and D. Hamer. 1988. Copper activates metallothionein gene transcription by altering the conformation of a specific DNA binding protein. Cell 55:705-717.

10. Giniger, E., S. M. Varnum, and M. Ptashne. 1985. Specific DNA binding of GAL4, a positive regulatory protein of yeast. Cell 40:767-774.

11. Hinnebusch, A. G. 1984. Evidence for translational regulation of the activator of general amino acid control in yeast. Proc. Natl. Acad. Sci. USA 81:6442-6446.

12. Johnson, P. F., and S. L. McKnight. 1989. Eukaryotic transcriptional regulatory proteins. Annu. Rev. Biochem. 58:799-839.

13. Johnston, M. 1987. A model fungal gene regulatory mechanism: the $G A L$ genes of Saccharomyces cerevisiae. Microbiol. Rev. 51:458-476.

14. Johnston, M. J., and J. Dover. 1987. Mutations that inactivate a yeast transcriptional regulatory protein cluster in an evolutionarily conserved DNA binding domain. Proc. Natl. Acad. Sci. USA 84:2401-2405.

15. Johnston, S. A., and J. E. Hopper. 1982. Isolation of the yeast regulatory gene GAL4 and analysis of its dosage effects on the galactose/melibiose regulon. Proc. Natl. Acad. Sci. USA 79: 6971-6975.
16. Marczak, J. E., and M. C. Brandriss. 1989. Isolation of constitutive mutations affecting the proline utilization pathway in Saccharomyces cerevisiae and molecular analysis of the PUT3 transcriptional activator. Mol. Cell. Biol. 9:4695-4704.

16a.Marczak, J. E., and M. C. Brandriss. Unpublished data.

17. Pan, T., and J. E. Coleman. 1990. GAL4 transcription factor is not a "zinc finger" but forms a $\mathrm{Zn}(\mathrm{II})_{2} \mathrm{Cys}_{6}$ binuclear cluster. Proc. Natl. Acad. Sci. USA 87:2077-2081.

18. Pfeifer, K., B. Arcangioli, and L. Guarente. 1987. Yeast HAP1 activator competes with the factor $\mathrm{RC} 2$ for binding to the upstream activation site UAS1 of the $C Y C l$ gene. Cell 49:9-18.

19. Ptashne, M. 1986. Gene regulation by proteins acting nearby and at a distance. Nature (London) 322:697-701.

20. Ptashne, M. 1988. How eukaryotic transcriptional activators work. Nature (London) 335:683-689.

21. Selleck, S. B., and J. Majors. 1987. Photofootprinting in vivo detects transcription-dependent changes in yeast TATA boxes. Nature (London) 325:173-177.

22. Selleck, S. B., and J. Majors. 1987. In vivo DNA-binding properties of a yeast transcription activator protein. Mol. Cell. Biol. 7:3260-3267.

23. Siddiqui, A. H., and M. C. Brandriss. 1988. A regulatory region responsible for proline-specific induction of the yeast PUT2 gene is adjacent to its TATA box. Mol. Cell. Biol. 8:4634-4641.

24. Siddiqui, A. H., and M. C. Brandriss. 1989. The Saccharomyces cerevisiae PUT3 activator protein associates with proline-specific upstream activation sequences. Mol. Cell. Biol. 9:47064712.

25. Sorger, P. K., and H. R. B. Pelham. 1988. Yeast heat shock factor is an essential DNA-binding protein that exhibits temperature-dependent phosphorylation. Cell 54:855-864.

26. Szczypka, M. S., and D. J. Thiele. 1989. A cysteine-rich nuclear protein activates yeast metallothionein gene transcription. Mol. Cell. Biol. 9:421-429.

27. Taylor, W. E., and E. T. Young. 1990. cAMP-dependent phosphorylation and inactivation of yeast transcription factor ADR1 does not affect DNA binding. Proc. Natl. Acad. Sci. USA 87:4098-4102.

28. Wang, S.-S., and M. C. Brandriss. 1986. Proline utilization in Saccharomyces cerevisiae: analysis of the cloned PUT1 gene. Mol. Cell. Biol. 6:2638-2645.

29. Wang, S.-S., and M. C. Brandriss. 1987. Proline utilization in Saccharomyces cerevisiae: sequence, regulation, and mitochondrial localization of the PUTI gene product. Mol. Cell. Biol. 7:4431-4440.

30. Wang, S. Y. (ed.). 1976. Photochemistry and photobiology of nucleic acids, vol. I. Academic Press, Inc., New York. 